\title{
Increase in Artemisia annua Plant Biomass Artemisinin Content and Guaiacol Peroxidase Activity Using the Arbuscular Mycorrhizal Fungus Rhizophagus irregularis
}

\author{
Erzsébet Domokos ${ }^{1 *}$, László Jakab-Farkas ${ }^{2}$, Béla Darkó ${ }^{1}$, Béla Bíró-Janka ${ }^{3}$, \\ Gyöngyvér Mara ${ }^{4}$, Csilla Albert ${ }^{5}$ and Adalbert Balog ${ }^{3 *}$
}

\begin{abstract}
'Department of Fundamental Pharmaceutical Sciences, Pharmaceutical Botany, Cell Biology and Microbiology, University of Medicine and Pharmacy of Târgu Mureș, Târgu Mureș, Romania, ${ }^{2}$ Department of Electrical Engineering, Sapientia Hungarian University of Transylvania, Cluj-Napoca, Romania, ${ }^{3}$ Department of Horticulture, Sapientia Hungarian University of Transylvania, Cluj-Napoca, Romania, ${ }^{4}$ Department of Bioengineering, Sapientia Hungarian University of Transylvania, Cluj-Napoca, Romania, ${ }^{5}$ Department of Food Science, Sapientia Hungarian University of Transylvania, Cluj-Napoca, Romania
\end{abstract}

OPEN ACCESS

Edited by:

Andrea Genre,

Università degli Studi di Torino, Italy

Reviewed by:

Wolfgang Eisenreich,

Technische Universität München,

Germany

Andrea Moglia,

Università degli Studi di Torino, Italy

*Correspondence:

Erzsébet Domokos

domokos.erzsebet@umftgm.ro

Adalbert Balog

adalbert.balog@ms.sapientia.ro;

adalbert.balog@gmail.com

Specialty section:

This article was submitted to

Plant Microbe Interactions,

a section of the journal

Frontiers in Plant Science

Received: 17 January 2018

Accepted: 28 March 2018

Published: 13 April 2018

Citation:

Domokos E, Jakab-Farkas L, Darkó B, Bíró-Janka B, Mara G, Albert $C$ and Balog A (2018) Increase in Artemisia annua Plant Biomass Artemisinin Content and Guaiacol Peroxidase Activity Using the Arbuscular Mycorrhizal Fungus Rhizophagus irregularis. Front. Plant Sci. 9:478. doi: 10.3389/fpls.2018.00478
The main objective of this study was to investigate Artemisia annua plant property variations in terms of plant biomass, glandular trichome numbers, artemisinin production and Guaiacol peroxidase (GPOX) activity when plants are in mutualism with AMF. According to the results, $A$. annua mutualism with AMF significantly increased the most important and pharmaceutically relevant factors of fresh and dry plant biomass. This increase, especially in the biomass of plant herba (leaves), was 30\% higher during the vegetation period and remained high (29\% higher than for control) when plants were harvested at the end of the vegetation period. Similar differences in dry biomass were also detected. Glandular trichomas numbers increased by $40 \%$, and the artemisinin content by $17 \%$ under AMF colonization. No effects due to AMF on chlorophyll variations were detected, while GPOX enzyme concentrations increased significantly under AMF colonization. Altogether the Artemisia plant properties with high pharmaceutically importance (fresh and dry biomass of leaves and artemisinin, number of trichomes and the artemisinin content) were significantly improved by AMF, the application in Artemisia cultivation can be an effective and cheap method. The high GPOX activity under AMF colonization indicate an enhanced oxidative stress alleviation, therefore a higher resistance to water deficiency, mechanisms important under climate conditions with low water supply where Artemisia is usually cultivated.

Keywords: AMF, plant-fungus interactions, plant biomass, glandular trichome, stress proteins, artemisinin content

\section{INTRODUCTION}

In developing nations, malaria is one of the most predominant parasitic infections and the 10th overall cause of death. It is estimated that it will remain at that level until 2030 (Mathers and Loncar, 2006; Ikram and Simonsen, 2017; Ikram et al., 2017). According to the World Health Organization (WHO and UNICEF, 2005), more than 380 million cases of malaria occur annually, being responsible for more than 1 
million deaths in tropical and subtropical regions (Mathers and Loncar, 2006; Weathers et al., 2011). Artemisia annua L. produces plant compounds such as artemisinin, an effective sesquiterpene lactone in malaria treatment, and also in combating other parasitic diseases, certain viral infections and neoplasms (Weathers et al., 2011; Suberu et al., 2013; Stefanache et al., 2016; Ikram and Simonsen, 2017). Based on previous research, the most representative phenolic compounds in A. annua are flavones and their glycosides (luteolin, luteolin-7glucoside, apigenin), flavonols and their glycosides (kaempferol, quercetin, isoquercitrin, rutin, patuletin), coumarins (coumarin, 6,7-dimethoxy-coumarin), and phenolic acids (ferulic acid) (Ivanescu et al., 2010; Stefanache et al., 2016; Ikram et al., 2017). As the compounds in Artemisia plants are in short supply, research on their production and its quantity in plants is of high commercial and medical value - as are low cost methods for drug delivery - moreover its cultivation are practiced under low water supply (Weathers et al., 2011). Although several studies have investigated the artemisinin production of Artemisia plants under different growing systems (Jelodar et al., 2014; Pulice et al., 2016), only a few of them have investigated the effect of arbuscular mycorrhizal funguses (AMFs) effects on these plants (Chaudhary et al., 2008; Awasthi et al., 2011). According to these studies, plants inoculated with Glomus macrocarpum and Glomus fasciculatum presented an increase in biomass (fresh and dry weight of shoot), concentration of specific nutrients $(\mathrm{P}, \mathrm{Zn}$, and $\mathrm{Fe})$ in shoots, and in artemisinin and essential oil content of plants (Chaudhary et al., 2008). Experiment using Glomus mosseae and Bacillus subtilis together indicated similar results regarding the growth, biomass and the content of artemisinin in A. annua plants (Awasthi et al., 2011). Some AMF species, such as Rhizophagus irregularis N.C. Schenck and G.S. Sm. (Glomerales: Glomeraceae), are capable to form mutualistic relationship with a wide range of plant species (Kloppholz et al., 2011). Although many of the processes involved in these mutualistic relationships are still unclear, it is known that plant fungus interaction has a positive influence on plants nutrition (absorption of phosphorus and microelements), water management and defense systems (Odebode and Salami, 2004; Hempel et al., 2009; Jiang et al., 2015). Recent studies have also revealed that mutualism is a genetically coordinated recognition process in which synchronized signaling pathways occur between the partners. During mutualism $R$. irregularis produces specific effector proteins that mediate adaptation of the fungus to the conditions present in the root-system (Kloppholz et al., 2011). Another protein secreted by $R$. irregularis, SP7, interacts with the pathogenesis-related transcription factor ERF19 of the plant (Kloppholz et al., 2011). Overexpression of some ERF transcription factors are involved in physiological processes that induce plant resistance to several biotic and abiotic stresses (Lee et al., 2004; Zhang et al., 2009). Little is known about how artemisinin and its metabolites are influenced throughout plant development and in relation to leaf trichomes (Weathers et al., 2006, 2011). During the previous experiments, trichomes densities were analyzed in three types of leaves, in floral buds and flowers, during three developmental stages: vegetative, floral budding, and full flower. According to the results, expression levels in the leaves of early pathway genes, HMGR, PFS, DXS, and DXR has not been correlated with either artemisinin or its precursors. Later pathway genes as $A D S$ and $C Y P$, however, has strong correlation with artemisinin precursor, DHAA, in leaf tissues. A close correlation between artemisinin levels and leaf trichome density was also detected (Weathers et al., 2011). While the A. annua cultivation are usually practiced under low water supply conditions, the role of some antioxidant enzyme activation as GPOX under AMF colonization are of high importance. No similar studies on Artemisia were made until now. Very recent studies on carob plants (Ceratonia siliqua L.) however demonstrated that AM colonized plants suffered less drought stress because AMF colonization improved plants water status by activating antioxidant enzymes (GPOX) (Essahibi et al., 2018). According to these in the present study, the main goals were to investigate plant property variations regarding plant biomass, glandular trichome numbers, artemisinin production, and antioxidant enzyme activity of $A$. annua plants when these plants are in mutualism with AMF. Our aim was to test the following hypotheses:

(1) Mutualism of A. annua plants with AMF R. irregularis can increase plant herb biomass.

(2) Plant glandular trichome numbers producing artemisinin can also be increased, thus the quantity of plant compounds (i.e., artemisinin) can also be improved under mutualism with $R$. irregularis.

(3) Antioxidant enzyme activity [Guaiacol peroxidase (GPOX)] may increase under AMF colonization.

\section{MATERIALS AND METHODS}

\section{Plant Material and Mycorrhizal Inoculation}

For this study, seeds of $A$. annua cultivar A-3, from Anamed (Winnenden, Germany) were used. A. annua A-3 is a lateflowering hybrid, usually maturing from March to October. It is a high yielding clone that was specially bred to have 20 times the amount of artemisinin found in the wild form. Seeds were germinated in March on peat disks with coconut fiber (Anamed, Germany), under the following laboratory conditions: a temperature of $22^{\circ} \mathrm{C}$, air humidity of $40 \%, 16 \mathrm{~h}$ light and $8 \mathrm{~h}$ dark photoperiod, $10 \mathrm{ml}$ water/disk/day (Figure 1A). The 14 day-old seedlings (in the 2 leaf stage) were transplanted in pots (with $50 \mathrm{~cm}$ length, 19.5 height, and $16.3 \mathrm{~cm}$ width) containing sterile commercial peat (Blondy Romania SRL) (Figure 1B). This growing system was chosen because other research has shown that the effect of $R$. irregularis under open field condition is low or even unobservable, and that the successful establishment of mutualistic interaction with plant roots in field soils depends on the indigenous species present, the species being introduced, and its placement in the soil (Hepper et al., 1988; Engelmoer et al., 2014). When the seedlings reached the 5-6 leaf stage (about 35 day-old seedlings) preparation was started for inoculation with AMF spores following the standard practice. The seedlings were placed separately in $4 \mathrm{l}$ pots with sterile peat and were 


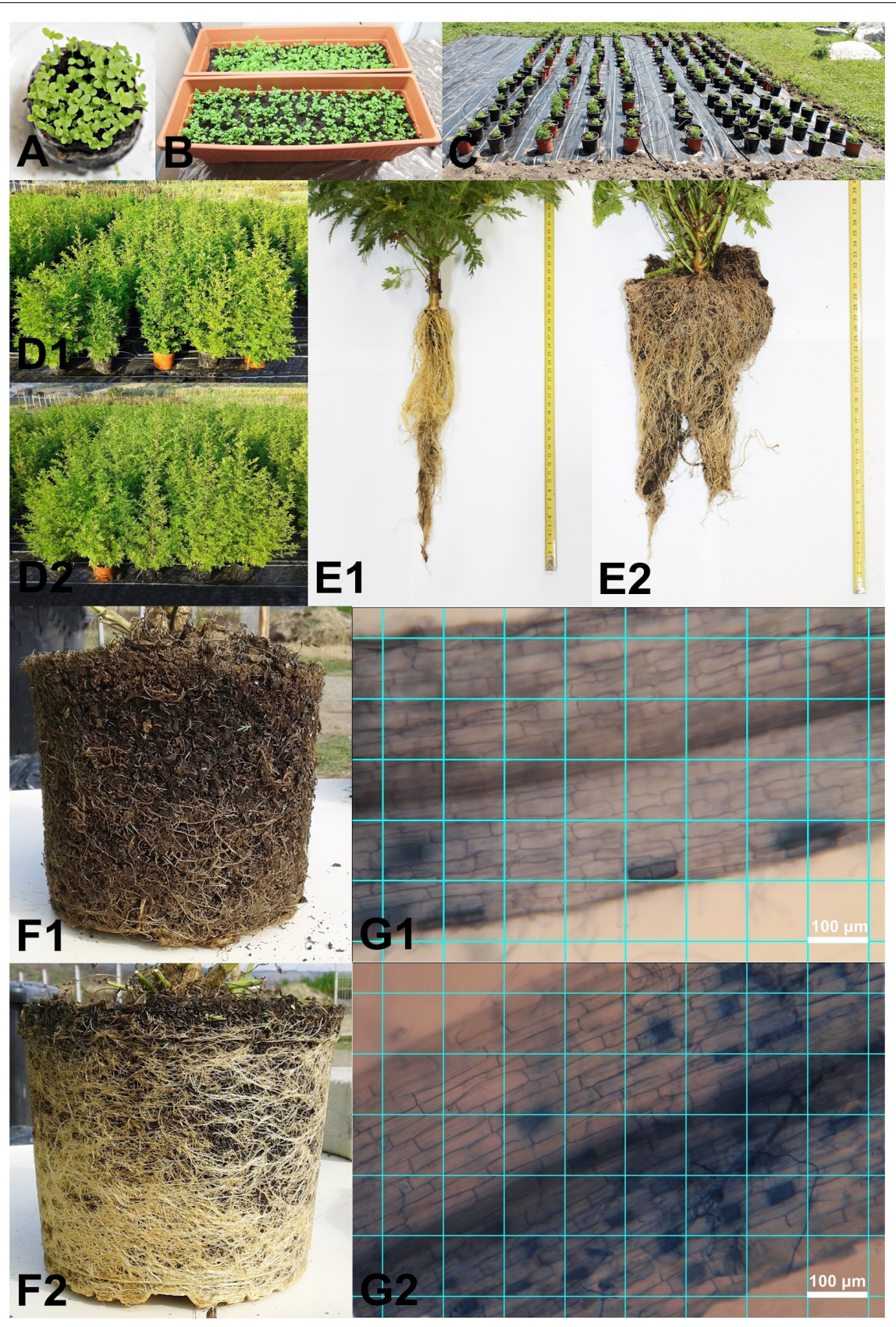

FIGURE 1 | Germinating seeds (A), young plants under lab growing conditions (B), field experiment set-up with blocks (C), control (D1) and AMF inoculated plants (D2) under vegetation period, root without AMF (E1) and root colonized with AMF (E2) at the start of the experiment, root without AMF (F1) and root colonized with AMF (F2) at the middle of the experiment in July and image greed of root colonization without AMF (G1) and with AMF (G2) (Image J Image Processing and Analysis in Java version 1.51j8) (Photos by Erzsébet Domokos).

watered with $400 \mathrm{ml}$ water/day. The inoculation was made with the AMF spores of $R$. irregularis purchased commercially (Italpollina SPA, Italy). Because the product of AMF spores was supplied as a high concentrated powder (1400 spores/g), $0.5 \mathrm{~g}$ mycorrhizal inoculum was added to each pot after dissolving in $400 \mathrm{ml}$ water. The pots were not watered in the first $24 \mathrm{~h}$ after the AMF treatment. During this period the mutualistic relationship between the plant and AMF could be established.
In the subsequent 3 weeks the plants inoculated with AMF were kept separate to those without AMF treatment in a greenhouse for accommodation to daylight.

\section{Experimental Design}

The field experiment was conducted in Corunca, Mureş County $\left(46^{\circ} 31^{\prime} 18.18^{\prime \prime} \mathrm{N}\right.$ and $\left.24^{\circ} 35^{\prime} 53.78^{\prime \prime} \mathrm{E}\right)$. The plants were moved outside from the greenhouse in early June in pots of 201 
containing sterile peat moss (only dead fibrous materials). This was done in order to test only the effect of AMF on Artemisia plants since under open field conditions the effect of AMF are highly confoundable because of other synergic fungus and bacteria species (Engelmoer et al., 2014). An experimental area of $500 \mathrm{~m}^{2}$ had previously been covered with a plastic wrap to avoid contact between the root system of the experimental plants and the soil. A total of 200 plants (100 plants inoculated with AMF and 100 control plants, without AMF treatment) were randomly arranged in a double nested block design. The experiment consisted of eight blocks, each of them comprising 25 plants. Inside each block, the space between the pots in each row was $30 \mathrm{~cm}$, and between the rows $50 \mathrm{~cm}$. The distance between the blocks was $1 \mathrm{~m}$. The entire system was connected to an automatic drip irrigation source (Figures 1C,D1,D2). The irrigation was performed with $400 \mathrm{ml}$ of water/pot daily during June and October, and $800 \mathrm{ml} /$ pot daily during July and August. The standard nutrients ( $\mathrm{N} 250, \mathrm{P} 50, \mathrm{~K} 250$, $\mathrm{Ca} 100, \mathrm{Mg} \mathrm{30}, \mathrm{Fe} 2, \mathrm{Mn} \mathrm{0.3,} \mathrm{B} \mathrm{0.3,} \mathrm{Cu} \mathrm{0.1,} \mathrm{Zn} 0.26$ ppm) were provided automatically every 2 nd week to consistently maintain the treatment regime for both AMF-treated and control plants.

\section{Root Colonization Analyses}

The AMF colonization was evaluated on three randomly selected plants/treatment/block (12 plants inoculated with AMF, and 12 control plants) at early vegetation period 1 week after inoculation (Figures 1E1,E2) and in the full vegetation period (8 week old plants) (Figures 1F1,F2). A random sub-sample of approximately 150 root fragments (of $1 \mathrm{~cm}$ length) per plant was collected. The roots were cleared in $2.5 \% \mathrm{KOH}\left(90^{\circ} \mathrm{C}\right)$ for $45 \mathrm{~min}$, acidified with $1 \% \mathrm{HCl}$ for $15 \mathrm{~min}$, stained with $0.05 \%$ Trypan Blue in acid glycerol $\left(90^{\circ} \mathrm{C}\right)$ for $45 \mathrm{~min}$, and then stored in acid glycerol (Koske and Gemma, 1989). From each subsample, 10 randomly selected root fragments were mounted in glycerine on slides. The fragments were aligned parallel to the long axis of the slide. Pictures from the root fragments were taken at 200x magnification by a photographic camera (Canon EOS 1100D, Taiwan) mounted on a microscope (Ceti Topic-T, Belgium). One picture, at 200x magnification, covered a field of view with a length of $0.882 \mathrm{~mm}$, and a width of $0.588 \mathrm{~mm}$. For the root fragment, five pictures were obtained. The distance between the pictures was constant for each sub-sample. In the few cases, when the root fragments were too wide to fit into the field of view of the camera, pictures were taken in two width portions (Figures 1G1,G2).

\section{Plant Biomass Measurements}

All plants were harvested until the late vegetative stage (in October). The fresh and dry weight of herba (healthy green leaves only) and roots were measured for 72 randomly harvested plants (9 plants/block). This was done in July at the full vegetation stage of all plants. Besides these measurements, the fresh weight of herba and roots for all remaining harvested plants used for the experiment was also determined. Before measurements were made, the plant leaves and roots were washed and dried on blotting paper. After fresh weight measurements, both the shoots and the roots were dried for 3 weeks under shade at an ambient temperature $\left(20^{\circ} \mathrm{C}\right)$ and analyses repeated on the dry plants.

\section{Glandular Trichome Density Assessment}

For the determination of glandular trichome density from the upper epidermis of the leaf, a scanning electron microscope (SEM) was used (JEOL JSM-5200, Japan). The SEM was utilized in order to achieve small magnification, high contrast micrographs using secondary electrons at $20 \mathrm{~mm}$ working distance, with 1 of $5 \mathrm{kV}$ accelerating voltage as function of sample charging. The fresh leaves of A. annua were collected at the full vegetation stage in July and were immediately subjected to analysis with the SEM. For the analysis 10 leaves of 10 randomly selected plants from each block were collected (a total of 80 plants sampled and 400 leaves/treatment analyzed). This represented almost completely the total upper leaves numbers of the plants. The youngest terminal leaf, of about $5 \mathrm{~cm}$ in length, on each plant was removed for sampling. The leaf surface was analyzed by starting with a fragment from the leaf mounted on a scanning surface in the form of a square with $0.6 \mathrm{~cm}$ sides (Figure 2). The number of glandular trichomes were counted with the multi-point tool, while the scanned leaf surface was measured with the polygon selection tool. Then the leaf surface was determined and the number of trichomes reported and averaged in $\mathrm{mm}^{2}$.

\section{Artemisinin Concentration Assessment}

A randomly selected 10 plants from each block were used to test the artemisinin content under AMF inoculation and

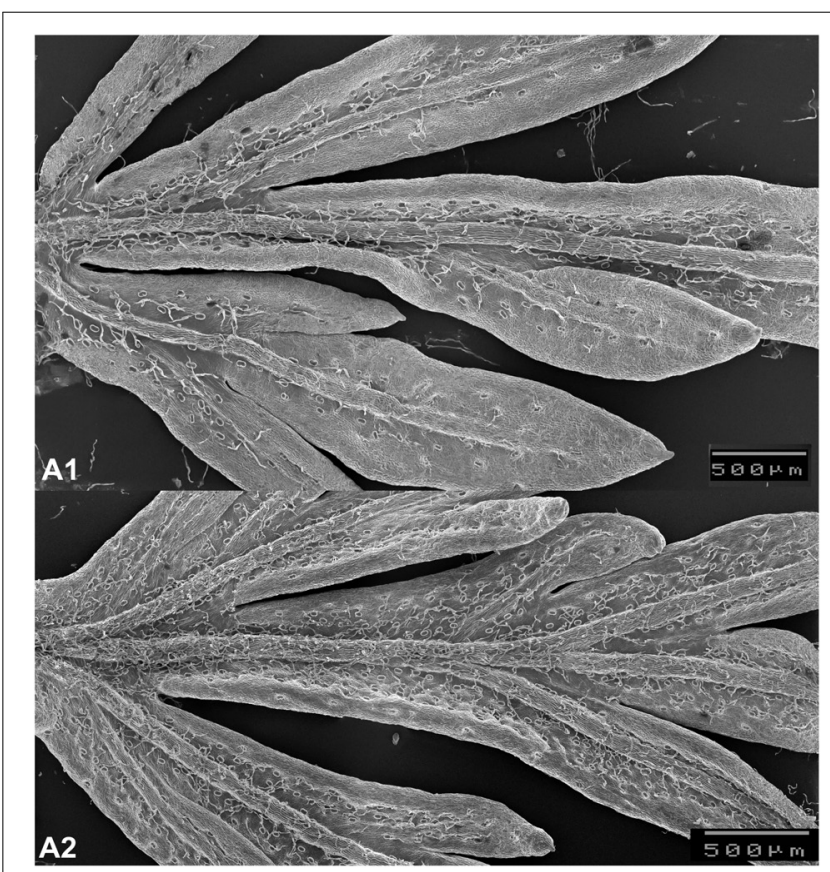

FIGURE 2 | Glandular trichomes on leaves without AMF (A1) and with AMF (A2) (scanning electron microscope, JEOL JSM-5200) image (Photos by László Jakab-Farkas). 
control. Standard artemisinin was purchased from Sigma-Aldrich (Germany). Artemisinin was extracted by refluxing $100 \mathrm{~g}$ of dry leaves with hexane at $75^{\circ} \mathrm{C}$ for $1 \mathrm{~h}$. The hexane was then evaporated under a vacuum and the samples reconstituted in $10 \mathrm{ml}$ acetonitrile then filtered through $0.45 \mu \mathrm{m}$ syringe filters. The HPLC analyses were performed with Agilent Infinity 1260 on Zorbax Eclipse Plus C18 (100 $\mathrm{mm} \times 3,5 \mathrm{~mm})$, column and detection was conducted at $210 \mathrm{~nm}$ wavelength. The acetonitrile: water $65: 35 \%(\mathrm{v} / \mathrm{v})$, was used as a mobile phase with $0.6 \mathrm{ml} / \mathrm{min}$ flow rate (Lapkin et al., 2009). The protocol developed by Lapkin et al. (2009) developed and validated an HPLC-RI method and optimized an HPLC-ELSD method. The gradient HPLC-UV method is widely recommended and used for quantification of artemisinin purity and amount in plant material. The calibration curve was constructed by plotting the peak area against the concentration $(1.5325,3.125,6.25,12.5$, $25 \mathrm{mg} / \mathrm{ml}$ ) of standard solutions. The determination coefficient $\left(\mathrm{R}^{2}\right)$ was 0.99985 .

\section{Assessment of Chlorophyll Concentrations}

The fresh leaves of 20 randomly selected plants (2-3 plants/block) were collected in August. For the spectrophotometric analysis, $0.5 \mathrm{~g}$ of fresh plant leaf per plant was used. Buffered $80 \%$ aqueous acetone was used for Chlorophyll $a$ and $b$ extraction according to Porra et al. (1989). The extraction was immediately followed by the absorbance measurement at 730, 664, and $647 \mathrm{~nm}$. Chlorophyll $a$ and $b$ content was calculated using the following equations (Porra et al., 1989).

$$
\begin{aligned}
& \text { Chl } a=12.25^{*} \mathrm{~A}^{664}-2.55^{*} \mathrm{~A}^{647} \\
& \text { Chl } b=20.31^{*} \mathrm{~A}^{647}-4.91^{*} \mathrm{~A}^{664}
\end{aligned}
$$

\section{Guaiacol Peroxidase (GPOX) Enzyme Extraction and Activity Assays}

Leaf samples used for enzyme analyses were collected from both AMF-treated and control plants on July with full vegetation period, before flowering. Samples were collected randomly from 20 AMF-treated and 20 control plants using the fifth leaves from the top of each plant and counted to have a $500 \mathrm{mg}$ sample/plant. Samples were kept at $-20^{\circ} \mathrm{C}$ until the enzyme extraction and activity assays. For extraction, $200 \mathrm{mg}$ of frozen leaves were homogenized in $1 \mathrm{ml}$ of QB buffer [100 mM $\mathrm{KPO}_{4}$ (pH 7.8), 1 mM EDTA, 1\% Triton X-100, 10\% glycerol, 1 mM DTT (added before use), distilled water], pH 7.8 using a FastPrep Instrument high-speed benchtop homogeniser (MP Biomedicals). The homogenate was centrifuged at $1,000 \mathrm{~g}$ for $30 \mathrm{~min}$ at $4^{\circ} \mathrm{C}$, and the supernatant collected. Protein concentration of the enzyme extract was determined by the Bradford method (Bradford, 1976). The activity of GPOX was determined spectrophotometrically at room temperature $\left(20-25^{\circ} \mathrm{C}\right)$, at a wavelength of $480 \mathrm{~nm}$ in a reaction mixture $(1 \mathrm{ml})$ containing $0.2 \mathrm{mM}$ phosphate buffer ( $\mathrm{pH}$ 7.5), $20 \mathrm{mM} \mathrm{H} \mathrm{H}_{2}$ and $20 \mathrm{mM}$ guaiacol $25 \mu \mathrm{l}$ of crude protein extract. Enzyme activity was determined according to Cavalcanti et al. (2004). One unit of GPOX activity was defined as the amount of enzyme producing $1 \mu \mathrm{mol}$ of tetraguaiacol per minute. Specific activity was expressed in $U / \mu g$ protein.

\section{Data Analyses}

The percentage of root colonization by AMF was determined using the program Image Image Processing and Analysis in Java version 1.51j8 (National Institute of Mental Health, Bethesda, $\mathrm{MD}$, United States). On the image a grid of lines was created with an area per point of $0.1 \mathrm{~mm}^{2}$. The points were counted in the following categories: with arbuscules, with vesicles, with hyphae only, with fungal material (one or more types of fungal material), with no fungal material, all examined points (Figures 1G1,G2). The arbuscular colonization (AC) and the vesicular colonization (VC) were calculated by dividing the number of categories by the total number of examined points. The hyphal colonization (HC) was calculated as the proportion of points with fungal material (Figures 1G1,G2). Only proportional differences were calculated between AMF and control plants (no statistical analyses).

Count data obtained from plant biomass, glandular trichomes, and artemisinin content were first tested for normality of

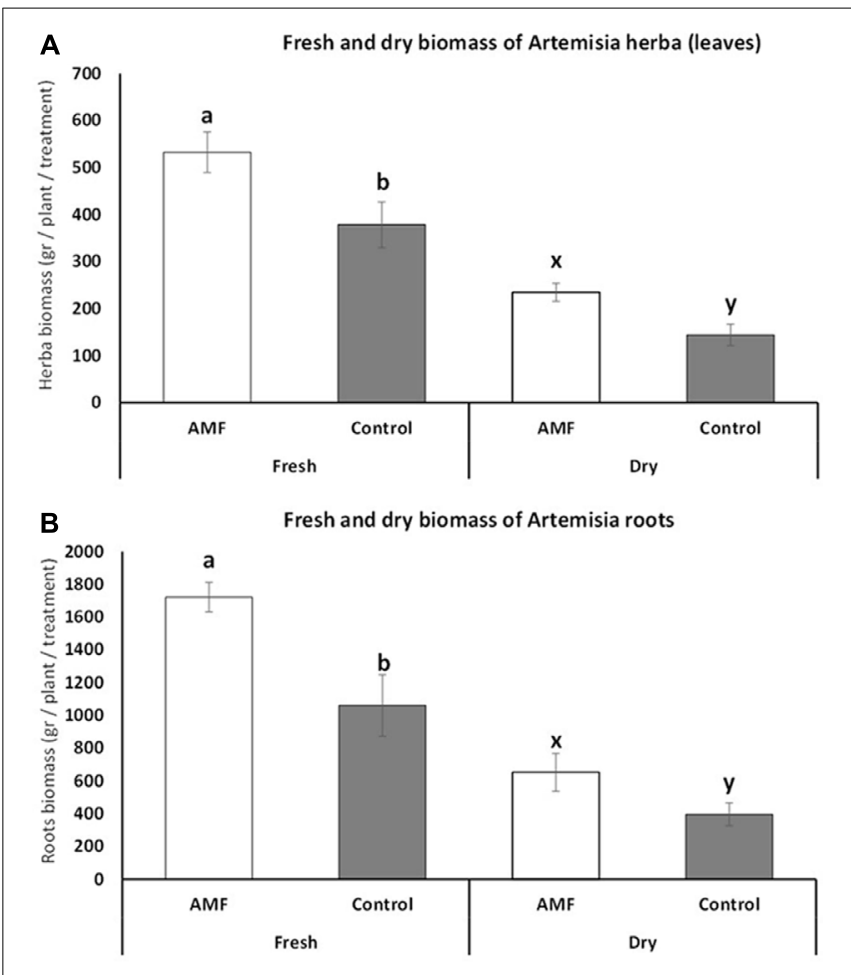

FIGURE 3 | Fresh and dry biomass of Artemisia annua herba (A) and roots (B) under AMF colonization (white bars) and control (without AMF) (gray bars) during the vegetation period (July). $t$-Test following the Student's $t$-distribution were used to compare variables. Different letters means statistical significant differences at $p<0.01$ level. Error bars $= \pm 1$ standard error. Data at harvest showed almost the same trend, therefore no additional figures were presented. 
errors (Kolmogorov-Smirnov) and for equality of variance (Levene's test). The plant biomass (fresh and dry herba and root) data, the glandular trichome data and the artemisinin content of leaves were normally distributed. Therefore $t$-tests, following the Student's $t$-distribution, were used to compare variables (fresh biomass between AMF and control, dry biomass between AMF and control for herba and roots separately). The glandular trichomas exact counts were made using the program ImageJ Image Processing and Analysis in Java version $1.51 \mathrm{j} 8$ (National Institute of Mental Health, Bethesda, MD, United States). Then the trichome data between AMF and control plants were compared with the $t$-test. Additionally, $t$-tests (again following the Student's $t$-distribution) were used to compare the content of artemisinin between AMF and control plants.

Chlorophyll and GPOX data did not meet the assumption of normality (Kolmogorov-Smirnov and Levene's test) therefore the Wilcoxon signed-rank test was used to compare concentrations between AMF inoculated plants and control plants. Results are presented in table. Linear correlation between the number of trichome and artemisinin concentration were made using Past Program and $r$ - and $p$-values calculated.

\section{RESULTS}

\section{Root Colonization Analyses}

Root colonization with AMF of Artemisia plants was successful in all cases. The observations on length and diameter for the young plants (Figures 1E,E1 control, E2 AMF colonized roots) and for the plants in full vegetation period (Figures 1F,F1 control, F2 AMF colonized roots) revealed that the root system of AMFtreated plants increased considerably. For the plants inoculated with AMF the AC was 34.75\% (SD: 10.50), the VC of $2.65 \%$ (SD: 1.29), and the HC of $50.37 \%$ (SD: 11.10).

\section{Plant Biomass Variation Under AMF Colonization}

The fresh biomass of plant herba at the full vegetation period increased significantly under AMF colonization $(t=3.37$, $p=0.007$ ) (Figure 3A). The average fresh biomass increase was $30 \%$ higher under AMF colonization, and the same trend was present at dry biomass with an average increase of $40 \%$ under AMF and significant differences when compared with the control $(t=3.99, p<0.001)$ (Figure 3A). Similarly, the root biomass of AMF plants was also higher for both fresh (average difference of $38 \%, t=6.38, p<0.001$ ) and dry biomass (average difference of $40 \%, t=6.39, p<0.001$ ) during the vegetation period (Figure $3 \mathbf{B}$ ). The herba and root fresh biomass at the end of the vegetation period (when the whole 200 plants total herba and root were compared) showed almost the same trend as the data for the vegetation period (average differences at leaf $31 \%$, $t=5.72, p<0.001$, average differences at $\operatorname{root} 39 \%, t=4, p<001)$. Again the dry biomass shoved the very similar results as during the vegetation period (average differences at leaf $37 \%, t=5.9$, $p<0.001$, average differences at root $39 \%, t=4.2, p<0.001)$.

\section{Glandular Trichome and Artemisinin Content Variation Under AMF Colonization}

Substantial increase (an average of 40.7\% higher number for AMF plants) and statistically significant differences in glandular trichome number were detected for plants under AMF colonization ( $t=3.63, p=0.003$ ) (Figure 4A). The content of artemisinin also increased significantly (with an average of $17 \%$ ) under AMF colonization compared to non-treated plants $(t=2.13, p=0.04)$ (Figure 4B). Highly significant positive linear correlation $(r=0.97, p=0.001)$ were observed between the number of trichomes and increase in artemisinin content.

\section{Chlorophyll and Guaiacol Peroxidase (GPOX) Concentration Variations Under AMF Colonization}

No differences between AMF and control plants were detected in chlorophyll (both chlorophyll a and chlorophyll b and its percentages). Significantly higher GPOX concentrations were detected on AMF plants comparing with control (Table 1).

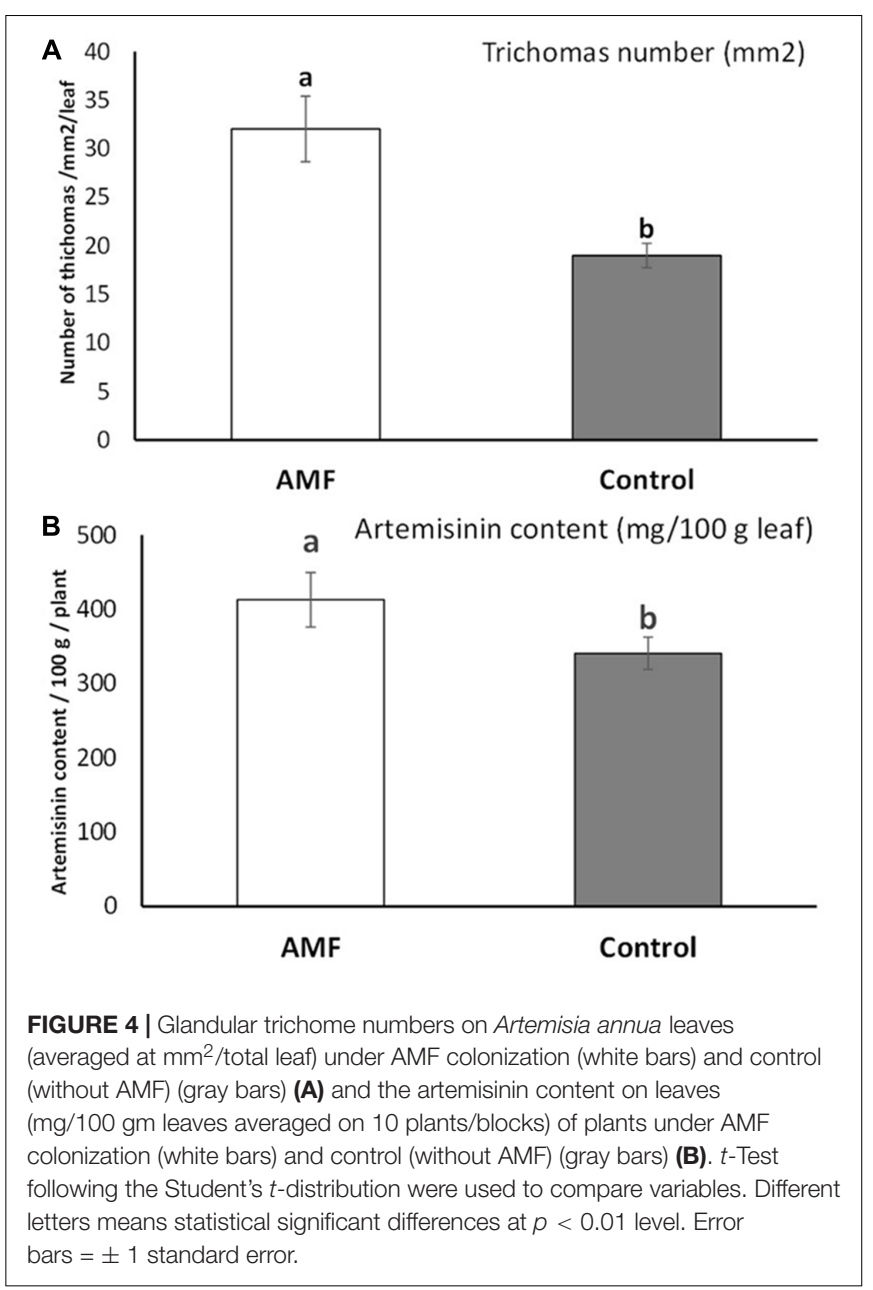




\section{DISCUSSION}

Based on these results, it is clear that $A$. annua mutualism with AMF significantly increased some important plant properties, such as the fresh and dry biomass of the plants, glandular trichomas numbers, and artemisinin content. Potential factors that may increase plant biomass under AMF colonization need to be considered as $R$. irregularis and other similar AMF species are obligatory biotrophs; in the absence of the host plant they only produce a tiny mycelium that aborts growth after a few days. In the presence of a compatible host plant, the fungus and plant exchange several secreted signals that initiate the symbiotic cellular program (Odebode and Salami, 2004; Bonfante and Genre, 2010; Bonfante and Requena, 2011). Similar studies also revealed that a significant increase in the biomass of $A$. annua was recorded on inoculation with Rhizophagus fasciculatus supplemented with $\mathrm{P}$ fertilizer. By analyzing the concentrations of mineral nutrients in the leaves of A. annua at the end of experiment, revealed that AMF inoculation significantly increased the concentrations of macroand micronutrients. Also a significant increase in the density of glandular trichome was recorded on AMF inoculated plants and an increase in concentration of artemisinin were detected in AMF plants compared to control. The study also revealed that AMF significantly increase the biomass production and accumulation of artemisinin (Giri, 2017).

The increase in plant biomass by mycorrhizal inoculation is probably due to reduction of leaf nitrogen, thereby increasing membrane stability and concentrations of essential inorganic nutrients such as $\mathrm{N}, \mathrm{P}$ and $\mathrm{K}$, as also reported by Selvakumar and Thamizhiniyan (2011). Several other plant properties may also be improved under AMF colonization - several control Artemisia plants became yellow at the end of the present experiments, while no discolorations on AMF plants were detected during the whole vegetation period. Decolouration can be explained by viral

TABLE 1 | Chlorophyll a and $b$ and guaiacol peroxidase (GPOX) concentration of AMF and control Artemisia annua plants.

\begin{tabular}{lll}
\hline & AMF & Control \\
\hline Chl.a & $N=20$ & $N=20$ \\
& Mean: 759.43 & Mean: 853.49 \\
& $F=1.0898$ & $p=0.28$ \\
Chl.b & Mean: 251.34 & Mean: 285.72 \\
& $F=1.01$ & $p=0.2$ \\
Chl a+b & Mean: 1010.8 & Mean: 1139.2 \\
& $F=1.07$ & $p=0.26$ \\
Chl a/b & Mean: 3.0207 & Mean: 2.9689 \\
& $F=1.14$ & $p=0.54$ \\
Guaiacol peroxidase (GPOX) & & \\
GPOX U/ml & Mean: 90.12 & Mean: 69.29 \\
GPOX U/ug & $F=3.67$ & $\boldsymbol{p}=\mathbf{0 . 0 4}$ \\
& Mean: 4.78 & Mean: 3.03 \\
& $F=4.11$ & $\boldsymbol{p}=\mathbf{0 . 0 4}$ \\
\hline
\end{tabular}

For statistical analyses the Wilcoxon signed-rank test were used. Bold values indicate statistical significant differences when $p<0.05$. infection of the control plants but also by low water quantity that might occur at plants with full vegetation stage, cultivated in pots. In other studies, it has also found that AMF colonization highly increased the jasmonate (MeJA) and oligogalacturonic acid, major plant defense signaling compounds conferring additional resistance and yield increase on vegetables such as tomato, potato, and soybean plants (Constabel et al., 1995). Similar research by Selvakumar and Thamizhiniyan (2011) has shown that in saline environment chili plants inoculated with AMF have a higher yield than control plants. Other researches revealed that $R$. irregularis, during its mutualism with vegetable plants, secretes in a very short time (after $20 \mathrm{~h}$ ) effector proteins that interact with transcription factors (e.g., ERF19) responsible for plant resistance against pathogens. These effector proteins remain active even after 14 days (Kloppholz et al., 2011). The increased resistance can be correlated with upregulation of certain defense-related genes, including PR1, PR2, PR4, Osmotin, and SAR8.2 (Lee et al., 2004; Zhang et al., 2009). The effect of AMF on Artemisia plants however has not been previously analyzed.

In our studies a highly significant correlation between trichome numbers and artemisinin content were detected. Similar researches revealed that mycorrhization stimulates the accumulation of artemisinin in plants by enhancing the number of glandular trichome on leafs and also by activating artemisinin synthesis responsible genes (Mandal et al., 2013, 2015). Other factors may also be of high importance in artemisinin production. Mycorrhization induces the methyl erythritol phosphate (MEP) pathway and enhances the level of isopentenyldiphosphate and dimethylallyldiphosphate, two important elements from which artemisinin is built of. It was observed that ibuprofen, an inhibitor of jasmonic acid, reduces the artemisinin concentration in shoots of non-mycorrhizal and mycorrhizal plants. Thus, this enzyme contributes to the mechanism of artemisinin production (Mandal et al., 2015; Ikram and Simonsen, 2017; Ikram et al., 2017). No effect of AMF on chlorophyll has been observed, while significant increase in GPOX activity was detected under AMF colonization by this experiment. The present study thus revealed that the high GPOX activity under AMF colonization may confer Artemisia plants a higher resistance to water deficiency, mechanisms important under climate conditions with low water supply where Artemisia is usually cultivated. According to other studies activation of GPOX were substantially increased in mycorrhizal carob plants under low water supply when experiments on carob plants were made, thereby showing enhanced resistance to oxidative stress induced by drought. Additionally, it has been showed that the effectiveness of AMF colonization in improving carob tolerance to drought was not related to an enhancement of the osmotic adjustment but to an improvement of the GPOX activities (Essahibi et al., 2018). These results are consistent with those previously reported in other plant species as olive, palm, and finger millet (Fouad et al., 2014; Benhiba et al., 2015; Tyagi et al., 2017), but no similar effect on Artemisia has been demonstrated until now.

Altogether the Artemisia plant properties with high pharmaceutically importance (fresh and dry biomass of leaves and artemisinin, number of trichomes, the artemisinin 
content and GPOX activity) were significantly improved by AMF, the application in Artemisia cultivation can be an effective and cheap method even under low water availability. The effect of AMF on other active ingredients however, has to be tested. The effect of AMF on A. annua plants are various and depends on several (probably environmental and biotic) factors that needs to be further considered in order to achieve a practically useful methods for AMF use in Artemisia production.

\section{AUTHOR CONTRIBUTIONS}

ED, LJ-F, BB-J, and AB performed the field experiments. ED and $\mathrm{BD}$ performed the root colonization analyses. ED and LJ-F performed the trichomas analyses. GM and CA performed the chlorophyll GPOX and Artemisinin analyses. ED performed the plant biomass analyses. $\mathrm{AB}$ and $\mathrm{ED}$ performed the data analyses. $\mathrm{AB}$ and $\mathrm{ED}$ prepared the figures and tables and wrote the main

\section{REFERENCES}

Awasthi, L. A., Bharti, N., Nair, P., Singh, R., Shukla, A. K., Gupta, M. M., et al. (2011). Synergistic effect of Glomus mosseae and nitrogen fixing Bacillus subtilis strain Daz26 on artemisinin content in Artemisia annua. Appl. Soil Ecol. 49, 125-130. doi: 10.1016/j.apsoil.2011.06.005

Benhiba, L., Fouad, M. O., Essahibi, A., Ghoulam, C., and Qaddoury, A. (2015). Arbuscular mycorrhizal symbiosis enhanced growth and antioxidant metabolism in date palm subjected to long-term drought. Trees 29, 1725-1733. doi: 10.1007/s00468-015-1253-9

Bonfante, P., and Genre, A. (2010). Mechanisms underlying beneficial plantfungus interactions in mycorrhizal symbiosis. Nat. Commun. 1:48. doi: 10.1038/ ncomms 1046

Bonfante, P., and Requena, N. (2011). Dating in the dark: how roots respond to fungal signals to establish arbuscular mycorrhizal symbiosis. Curr. Opin. Plant Biol. 14, 451-457. doi: 10.1016/j.pbi.2011.03.014

Bradford, M. M. (1976). A rapid and sensitive method for the quantitation of microgram quantities of protein utilizing the principle of protein-dye binding. Anal. Biochem. 72, 248-254. doi: 10.1016/0003-2697(76)90527-3

Cavalcanti, F. R., Oliveira, J. T. A., Martins-Miranda, A. S., Viégas, R. A., and Silveira, J. A. G. (2004). Superoxide dismutase, catalase and peroxidase activities do not confer protection against oxidative damage in salt-stressed cowpea leaves. New Phytol. 163, 563-571. doi: 10.1111/j.1469-8137.2004.01139.x

Chaudhary, V. L., Kapoor, R., and Bhatnagar, A. K. (2008). Effectiveness of two arbuscular mycorrhizal fungi on concentrations of essential oil and artemisinin in three accessions of Artemisia annua. Appl. Soil Ecol. 40, 174-181. doi: 10.1016/j.apsoil.2008.04.003

Constabel, C. P., Bergey, D. R., and Ryan, C. A. (1995). Systemin activates synthesis of wound-inducible tomato leaf polyphenol oxidase via the octadecanoid defense signaling pathway. Proc. Natl. Acad. Sci. U.S.A. 92, 407-411. doi: 10.1073/pnas.92.2.407

Engelmoer, D. J. P., Behm, J. E., and Toby Kiers, E. (2014). Intense competition between arbuscular mycorrhizal mutualists in an in vitro root microbiome negatively affects total fungal abundance. Mol. Ecol. 23, 1584-1593. doi: $10.1111 / \mathrm{mec} .12451$

Essahibi, A., Benhiba, L., Babram, M. A., Ghoulam, C., and Qaddoury, A. (2018). Influence of arbuscular mycorrhizal fungi on the functional mechanisms associated with drought tolerance in carob (Ceratonia siliqua L.). Trees 32, 87-97. doi: 10.1007/s00468-017-1613-8

Fouad, M. O., Essahibi, A., Benhiba, A., and Qaddoury, A. (2014). Effectiveness of arbuscular mycorrhizal fungi in the protection of olive plants against oxidative stress induced by drought. Span. J. Agric. Res. 12, 763-771. doi: 10.5424/sjar/ 2014123-4815

Giri, B. (2017). "Mycorrhizal fungus Rhizophagus fasciculatus promotes artemisinin accumulation in Artemisia annua," in Paper Presented at the manuscript text. $\mathrm{AB}$ edited and corrected the language of the manuscript. All authors reviewed the manuscript and agreed with submission.

\section{FUNDING}

This work was supported by the scholarship ELTE/7252/1/2017 from the Ministry of Human Capacities (Hungarian Government) and the Eötvös Loránd University, for the 2016/2017 academic year.

\section{ACKNOWLEDGMENTS}

The authors are grateful to Mr. David Speight and TransEnglish for English language corrections. They thank reviewers for their constructive comments.

Tropentag, Future Agriculture: Socio-Ecological Transitions and Bio-Cultural Shifts, Bonn.

Hempel, S., Stein, C., Unsicker, S. B., Renker, C., Auge, H., and Weisser, W. W. (2009). Specific bottom-up effects of arbuscular mycorrhizal fungi across a plant-herbivore-parasitoid system. Oecologia 160, 267-277. doi: 10.1007/ s00442-009-1294-0

Hepper, C. M., Azcon-Aguilar, C., Rosendahl, S., and Sen, R. (1988). Competition between three species of Glomus used as spatially separated introduced and indigenous mycorrhizal inocula for leek (Allium porrum L.). New Phytol. 110, 207-215. doi: 10.1111/j.1469-8137.1988.tb00254.x

Ikram, N. K. B., Beyraghdar Kashkooli, A., Peramuna, A. V., van der Krol, A. R., Bouwmeester, H., and Simonsen, H. T. (2017). Stable production of the antimalarial drug artemisinin in the moss Physcomitrella patens. Front. Bioeng. Biotechnol. 5:47. doi: 10.3389/fbioe.2017.00047

Ikram, N. K. B. K., and Simonsen, H. T. (2017). A review of biotechnological artemisinin production in plants. Front. Plant Sci. 8:1966. doi: 10.3389/fpls. 2017.01966

Ivanescu, B., Vlase, L., Corciova, A., and Lazar, M. I. (2010). HPLC-DAD-MS study of polyphenols from Artemisia absinthium. A. annua and A. vulgaris. Chem. Nat. Compd. 46, 468-470. doi: 10.1007/s10600-010-9648-8

Jelodar, N. B., Bhatt, A., Mohamed, K., and Keng, C. L. (2014). New cultivation approaches of Artemisia annua L. for a sustainable production of the antimalarial drug artemisinin. J. Med. Plants Res. 8, 441-447. doi: 10.5897/ JMPR11.1053

Jiang, J. H., Lee, Y. I., Cubeta, M. A., and Chen, L. C. (2015). Characterization and colonization of endomycorrhizal Rhizoctonia fungi in the medicinal herb Anoectochilus formosanus (Orchidaceae). Mycorrhiza 25, 431-445. doi: 10.1007/ s00572-014-0616-1

Kloppholz, S., Kuhn, H., and Requena, N. (2011). A secreted fungal effector of Glomus intraradices promotes symbiotic biotrophy. Curr. Biol. 21, 1204-1209. doi: 10.1016/j.cub.2011.06.044

Koske, R. E., and Gemma, J. N. A. (1989). A modified procedure for staining roots to detect VA mycorrhizas. Mycol. Res. 92, 486-505. doi: 10.1016/S09537562(89)80195-9

Lapkin, A. A., Walker, A., Sullivan, N., Khambay, B., Mlambo, B., and Chemat, S. (2009). Development of HPLC analytical protocols for quantification of artemisinin in biomass and extracts. J. Pharm. Biomed. Anal. 49, 908-915. doi: 10.1016/j.jpba.2009.01.025

Lee, J. H., Hong, J. P., Oh, S. K., Lee, S., Choi, D., and Kim, W. (2004). The ethylene-responsive factor like protein 1 (CaERFLP1) of hot pepper (Capsicum annuum L.) interacts in vitro with both GCC and DRE/CRT sequences with different binding affinities: possible biological roles of CaERFLP1 in response to pathogen infection and high salinity conditions in transgenic tobacco plants. Plant Mol. Biol. 55, 61-81. doi: 10.1007/s11103-0040417-6 
Mandal, S., Evelin, H., Giri, B., Singh, V. P., and Kapoor, R. (2013). Arbuscular mycorrhiza enhances the production of stevioside and rebaudioside-A in Stevia rebaudiana via nutritional and non-nutritional mechanisms. Appl. Soil Ecol. 72, 187-194. doi: 10.1016/j.apsoil.2013.07.003

Mandal, S., Upadhyay, S., Wajid, S., Ram, M., Jain, D. C., Singh, V. P., et al. (2015). Arbuscular mycorrhiza increase artemisinin accumulation in Artemisia annua by higher expression of key biosynthesis genes via enhanced jasmonic acid levels. Mycorrhiza 25, 345-357. doi: 10.1007/s00572-014-0614-3

Mathers, C. D., and Loncar, D. (2006). Projections of global mortality and burden of disease from 2002 to 2030. PLoS Med. 3:e442. doi: 10.1371/journal.pmed. 0030442

Odebode, A. C., and Salami, A. O. (2004). Biochemical contents of pepper seedlings inoculated with Phytophthora infestans and arbuscular mycorrhiza. J. Agric. Sci. 49, 251-257. doi: 10.2298/JAS0402251O

Porra, R. J., Thompson, W. A., and Kriedemann, P. E. (1989). Determination of accurate extinction coefficients and simultaneous equations for assaying chlorophylls a and b extracted with four different solvents: verification of the concentration of chlorophyll standards by atomic absorption spectroscopy. Biochim. Biophys. Acta 975, 384-394. doi: 10.1016/S0005-2728(89)80347-0

Pulice, G., Pelaz, S., and Matías-Hernández, L. (2016). Molecular farming in Artemisia annua, a promising approach to improve anti-malarial drug production. Front. Plant Sci. 7:329. doi: 10.3389/fpls.2016.00329

Selvakumar, G., and Thamizhiniyan, P. (2011). The effect of the arbuscular mycorrhizal (AM) fungus Glomus intraradices on the growth and yield of chilli (Capsicum annuum L.) under salinity stress. World Appl. Sci. J. 14, 1209-1214.

Stefanache, C. P., Bujor, O. C., Necula, R., Danila, D., and Ciocârlan, N. (2016). Phenolic Content of Artemisia Annua L. From Natural Habitats in Republic of Moldova - ProQuest. Available at: https://search.proquest.com/openview/ 0a6465eddb891c1456215087ced38ed3/1?pq-origsite $=$ gscholar $\& \mathrm{cbl}=676306$ [Accessed December 30, 2017].

Suberu, J., Song, L., Slade, S., Sullivan, N., Barker, G., and Lapkin, A. A. (2013). A rapid method for the determination of artemisinin and its biosynthetic precursors in Artemisia annua L. crude extracts. J. Pharm. Biomed. Anal. 84, 269-277. doi: 10.1016/j.jpba.2013.06.025
Tyagi, J., Varma, A., and Pudake, R. N. (2017). Evaluation of comparative effects of arbuscular mycorrhiza (Rhizophagus intraradices) and endophyte (Piriformospora indica) association with finger millet (Eleusine coracana) under drought stress. Eur. J. Soil Biol. 81, 1-10. doi: 10.1016/j.ejsobi.2017. 05.007

Weathers, P. J., Arsenault, P. R., Covello, P. S., McMickle, A., Teoh, K. H., and Reed, D. W. (2011). Artemisinin production in Artemisia annua studies in planta and results of a novel delivery method for treating malaria and other neglected diseases. Phytochem. Rev. 10, 173-183. doi: 10.1007/s11101-0109166-0

Weathers, P. J., Elkholy, S., and Wobbe, K. K. (2006). Artemisinin: the biosynthetic pathway and its regulation in Artemisia annua, a terpenoid-rich species. In Vitro Cell. Dev. Biol. Plant 42, 309-317. doi: 10.1002/cbdv.2009 00313

WHO and UNICEF (2005). World Malaria Report. Switzerland: Marketing and Dissemination, World Health Organization.

Zhang, G., Chen, M., Li, L., Xu, Z., Chen, X., and Guo, J. (2009). Overexpression of the soybean GmERF3 gene, an AP2/ERF type transcription factor for increased tolerances to salt, drought, and diseases in transgenic tobacco. J. Exp. Bot. 60, 3781-3796. doi: 10.1093/jxb/erp214

Conflict of Interest Statement: The authors declare that the research was conducted in the absence of any commercial or financial relationships that could be construed as a potential conflict of interest.

The reviewer AM and handling Editor declared their shared affiliation.

Copyright (C) 2018 Domokos, Jakab-Farkas, Darkó, Bíró-Janka, Mara, Albert and Balog. This is an open-access article distributed under the terms of the Creative Commons Attribution License (CC BY). The use, distribution or reproduction in other forums is permitted, provided the original author(s) and the copyright owner are credited and that the original publication in this journal is cited, in accordance with accepted academic practice. No use, distribution or reproduction is permitted which does not comply with these terms. 